\title{
Bone Progenitors Produced by Direct Osteogenic Differentiation of the Unprocessed Bone Marrow Demonstrate High Osteogenic Potential In Vitro and In Vivo
}

\author{
Irene Ginis, ${ }^{1, *}$ Miron Weinreb, ${ }^{2}$ Natalie Abramov, ${ }^{1, \dagger}$ Doron Shinar, ${ }^{1}$ Shoshana Merchav, ${ }^{1}$ \\ Aharon Schwartz, ${ }^{1}$ and Mitchell Shirvan ${ }^{1, *}$
}

\begin{abstract}
Tissue-engineered bone grafts seeded with mesenchymal stem cells (MSCs) have been sought as a replacement for bone grafts currently used for bone repair. For production of osteogenic constructs, MSCs are isolated from bone marrow (BM) or other tissues, expanded in culture, then trypsinized, and seeded on a scaffold. Predifferentiation of seeded cells is often desired. We describe here bone progenitor cells (BPCs) obtained by direct osteogenic differentiation of unprocessed BM bypassing isolation of MSCs. Human BM aspirates were incubated for 2 weeks with a commonly used osteogenic medium $(\mathrm{OM})$, except no fetal calf serum, serum substitutes, or growth factors were added, because responding stem and/or progenitor cells were present in the BM milieu. The adherent cells remaining after the culture medium and residual BM were washed out, expressed high levels of bone-specific alkaline phosphatase (ALP) on their surface, demonstrated high ALP activity, were capable of mineralization of the intercellular space, and expressed genes associated with osteogenesis. These parameters in BPCs were similar and even at higher levels compared to MSCs subjected to osteogenic differentiation for 2 weeks. The yield of BPCs per $1 \mathrm{~mL} \mathrm{BM}$ was $0.71 \pm 0.39 \times 10^{6}$. In comparison, the yield of MSCs produced by adhesion of mononuclear cells derived from the same amount of BM and cultured in a commercial growth medium for 2 weeks was $0.3 \pm 0.17 \times 10^{6}$. When a scaffold was added to the BM-OM mixture, and the mixture was cultured in a simple rotational bioreactor; the resulting BPCs were obtained already seeded on the scaffold. BPCs seeded on scaffolds were capable of proliferation for at least 6 weeks, keeping high levels of ALP activity, expressing osteogenic genes, and mineralizing the scaffolds. Autologous rat BPCs seeded on various scaffolds were transplanted into critical-size calvarial defects. Six weeks after transplantation of polylactic acid/polyglycolic acid scaffolds, $76.1 \% \pm 18.3 \%$ of the defects were filled with a new bone, compared to $37.9 \% \pm 28.4 \%$ in the contralateral defects transplanted with the scaffolds without cells.
\end{abstract}

Key words: bioengineering; bone progenitors; calvaria; mesenchymal stem cells; scaffold

\section{Introduction}

I N THE CASE of the loss of large bone segments as a result of trauma or a tumor resection, the bone defect is usually filled with a porous scaffold or a bone graft is used. ${ }^{1}$ With recent progress in regenerative medicine, attempts have been made to seed scaffolds with adult mesenchymal stem cells (MSCs) found in the bone marrow (BM), fat, and other tissues (reviewed in Refs. ${ }^{2-5}$ ). Transplanted MSCs are assumed to undergo osteogenic differentiation in the bone environment or alternatively are predifferentiated before the transplantation. ${ }^{6}$

The low frequency of MSCs in BM requires expansion of isolated cells in vitro, which depends on preselected fetal calf serum (FCS). The differentiation potential of MSCs at later passages (40-50 population doublings) is often low due to MSC senescence, ${ }^{7}$ or tumor transformation. ${ }^{8}$

We hypothesized that osteogenic progenitors sought for tissue engineering bone grafts could be derived directly from BM without isolation of MSCs, but rather by treating

\footnotetext{
${ }^{1}$ Teva Pharmaceutical Industries LTD, Petach Tikva, Israel.

${ }^{2}$ School of Dental Medicine, Tel Aviv University, Tel Aviv, Israel.

*Present address: Macrocure, LTD, Petach Tikva, Israel.

†Present address: BrainStorm Cell Therapeutics LTD, Petach Tikva, Israel.
} 
of unprocessed BM with osteogenic factors. We assumed that osteogenic agonists would target all the cells in BM that are capable of osteogenic differentiation, whereas a part of these bone progenitor cells (BPCs) is lost during isolation of MSCs. In addition, osteogenic differentiation within unprocessed BM would be supported by growth factors and cytokines that are present in the BM milieu and by signals from adjacent cells.

We present here a method for derivation of BPCs directly from BM, and confirm the osteogenic character of these cells. When BPCs are produced in a rotational bioreactor in the presence of a scaffold, they populate the scaffold. We show that scaffolds seeded with BPCs during differentiation of unprocessed BM promote bone repair in vivo.

\section{Materials and Methods}

\section{Production of human BPCs}

Human BM aspirates were purchased from Lonza Inc. $\mathrm{BM}$ was mixed with an osteogenic medium (OM) [alphaMEM (Sigma), $0.2 \mathrm{mM}$ L-ascorbic acid-2-phosphate (Mg salt, n-hydrated; Fluka), $100 \mathrm{nM}$ dexamethasone (Sigma), 2 mM GlutaMAX ${ }^{\mathrm{TM}}$-I Supplement (Invitrogen), 100 units $/ \mathrm{mL}$ penicillin, $0.1 \mathrm{mg} / \mathrm{mL}$ streptomycin, and $0.25 \mathrm{mg} / \mathrm{mL}$ amphotericin B] at the ratio 1:5, plated onto 24-well plates or cell culture dishes (all from NUNC) precoated with $10 \mu \mathrm{g} /$ $\mathrm{mL}$ bovine fibronectin (Biological Industries), and cultured for 2 weeks in a cell incubator. No FCS was added to OM. In experiments with scaffolds, $0.2 \mathrm{~mL}$ BM was combined with $2.5 \mathrm{~mL} \mathrm{OM}$ and with a fibronectin-precoated scaffold in a 50-mL polypropylene tube with a filter cap (Techno Plastic Products AG). The tubes with the BM-OM mixture were placed on a HUG tube rotator (MRC) and rotated in the cell incubator at $6-10 \mathrm{rpm} / \mathrm{min}$ at an angle of $30^{\circ}$ for 2 weeks. After 1 week, half of the volume of each culture was replaced with a fresh OM, and incubation continued for another week. At 2 weeks, all cultures were washed 3 times with MEM-alpha, and BPCs adherent to the culture vessels or scaffolds (BPCs p0) were used for analysis. All $\mathrm{BPC}$ batches used in the experiments were derived from different BM donors.

\section{Production of rat BPCs}

$\mathrm{BM}$ from one femur/animal $(\sim 1 \mathrm{~mL})$ of 14-week-old Fischer344 inbred rats was flushed with $5 \mathrm{~mL}$ of OM. About $0.6 \mathrm{~mL}$ of the $\mathrm{BM} / \mathrm{OM}$ mixture was either seeded into a 24well plate or combined with $2.5 \mathrm{~mL} \mathrm{OM}$ and rotated with a fibronectin-precoated scaffold disc as described for human cells. Rat BPC cultures were completely washed after 1 week. At 2 weeks, the scaffolds were washed and used for transplantation.

\section{Isolation of human MSCs}

Human MSCs were isolated as described elsewhere, ${ }^{9}$ adopting the commonly used protocol. ${ }^{10}$ MSCs expressed all the main MSC markers, CD105, CD29, CD73, CD44, CD90, CD166, CD9, CD106, and CD146, and were negative for the hematopoietic cell marker CD $45 .{ }^{9}$ MSCs were capable of trilineage differentiation into osteoblasts, adipocytes, and chondrocytes. $^{9}$ All MSC batches used in the experiments were derived from different BM donors.

\section{Osteogenic differentiation of human MSCs}

Cells were plated at 3000 cells per $1 \mathrm{~cm}^{2}$ in GM overnight. Then, GM was replaced by the OM containing $10 \%$ heatinactivated FCS (Biological Industries). The OM was changed twice a week.

\section{Scaffolds}

Polylactic acid (PLA) (70/30 L, DL-PLA), polylactic/ polyglycolic acid copolymer (PLA/PGA) 50/50\%, and PLA/ceramics (70/30 L,DL-PLA compounded with $80 \%$ beta-tricalcium phosphate by mass) were purchased from Kensey Nash Corporation. PLA/PGA 75/25\% scaffolds used for animal studies and translucent PLA/PGA 50/50 scaffolds used for microscopic evaluation were a generous gift from Dr. Shulamit Levenberg, Technion, Haifa, Israel. All the scaffolds used in in-vitro studies were of 10-mm diameter and 1-2-mm thickness; the scaffolds used for animal studies were of 5-mm diameter and 2-mm thickness.

\section{Quantitation of MSCs and BPCs with Calcein-AM}

Cells were incubated with $5 \mu \mathrm{M}$ Calcein-AM (Invitrogen) in Dulbecco's modified Eagle's medium (DMEM) without phenol red in 24-well plates at $37^{\circ} \mathrm{C}$ for $30 \mathrm{~min}$. Cell fluorescence was read on the Synergy-BioTek plate reader at Ex/Em 485/ $530 \mathrm{~nm}$. For a standard curve, serial dilutions of MSC or BPC cell suspensions prestained with Calcein-AM were plated onto a 24-well plate and spun down to the bottom of the wells.

\section{Quantitation of BPCs growing on scaffolds with Alamar Blue}

Scaffolds seeded with cells were incubated with $10 \%$ Alamar Blue (Invitrogen) in the $\mathrm{OM}$ at $37^{\circ} \mathrm{C}$ for $2 \mathrm{~h}$. Fluorescence of the incubation medium was measured at Ex/Em 530/ $590 \mathrm{~nm}$. For a standard curve, serial dilutions of BPCs were plated in a 24-well plate and allowed to adhere overnight. Adherent cells were incubated with Alamar Blue in the presence of empty scaffolds. The exact cell numbers were determined with Calcein-AM after the Alamar Blue assay.

\section{Measurements of alkaline phosphatase activity}

Cells growing in a 24-well plate or on a scaffold were incubated with $1 \mathrm{~mL}$ alkaline lysis buffer for $1 \mathrm{~h}$ on ice. $100 \mu \mathrm{L}$ lysate combined with $400 \mu \mathrm{L}$ phosphatase substrate solution [20 $\mathrm{mg} / \mathrm{mL}$ of p-nitrophenol phosphate in $5 \mathrm{~mL}$ alkaline buffer solution diluted 1:3 with $\mathrm{ddH}_{2} \mathrm{O}$ ] was incubated at $37^{\circ} \mathrm{C}$ for $10 \mathrm{~min}$. The reaction was stopped with $500 \mu \mathrm{L}$ EDTA$\mathrm{NaOH}$ stop solution $\left[20 \mathrm{~g} \mathrm{NaOH}\right.$ plus $37.22 \mathrm{~g} \mathrm{Na}_{2}$ EDTA in $500 \mathrm{~mL} \mathrm{ddH} \mathrm{H}_{2} \mathrm{O}$ ]. The absorbance of the samples was read at $404 \mathrm{~nm}$. Serial dilutions of the dephosphorylated product, p-nitrophenol, were prepared for a standard curve. The results were expressed as nmol $\mathrm{p}-\mathrm{NP} / \mathrm{mL} / \mathrm{min} / 10,000$ live cells. All the reagents were from Sigma-Aldrich.

\section{Flow cytometry analysis of ALP expression}

BPCs or MSCs were incubated with saturating concentrations of anti-ALP antibodies (clone B478; R\&D) in an FACS buffer [2\% BSA (Sigma-Aldrich) and 2\% human blocking serum (Chemicon) in DMEM without phenol red (Sigma), $\mathrm{pH}$ 7.4] for $30 \mathrm{~min}$ on ice and then analyzed on FACSAria (BD). 


\section{Quantitation of calcium deposition}

Cells in a 24-well plate or on a scaffold were mixed with $250 \mu \mathrm{L} 0.5 \mathrm{~N} \mathrm{HCl}$. The lysates were shaken overnight at $4^{\circ} \mathrm{C}$ to extract calcium and then centrifuged at $13,000 \mathrm{rpm}$ for $2 \mathrm{~min}$. The assay was set up in 96-well plates using the Calcium Liquicolor kit (Stanbio Labs), according to the manufacturer's instructions.

\section{Fast Blue RR staining for ALP activity}

Cells growing in 24-well plates or on scaffolds were fixed with a mixture of 1:50 dilution of citrate solution (SigmaAldrich) and acetone at 2:3 ratio for $30 \mathrm{sec}$ and then stained with an alkaline phosphatase staining kit (Sigma-Aldrich), according to the manufacturer's instructions.

\section{Alizarin red staining for calcium deposits}

Cells growing in 24-well plates or on scaffolds were fixed with $4 \%$ paraformaldehyde for $15 \mathrm{~min}$ at room temperature, washed, and stained with $5 \mathrm{mg} / \mathrm{mL}$ Alizarin Red S (SigmaAldrich) for $1 \mathrm{~min}$.

\section{Microscopic evaluation}

Stained cells were analyzed using either bright-field or fluorescent microscopy (Zeiss AxioVert 40) and photographed with AxioCam MRC5.

\section{RNA isolation}

The RNeasy kit (Qiagen) was used to isolate RNA. BPCs growing on scaffolds were lyzed directly. RNase-free DNase (Qiagen) was used for DNase digestion. RNA was frozen at $-80^{\circ} \mathrm{C}$. Aliquots of RNA were thawed on ice, and RNA integrity was confirmed by electrophoresis in $2 \%$ agarose gel. The RNA yield from BPCs produced on PLA and PLA/ ceramic scaffolds was $9.8 \pm 5.0$ and $11.5 \pm 4.1 \mu \mathrm{g}$ per scaffold, respectively (mean $\pm \mathrm{SD} ; n=4$ ).

\section{Real-time polymerase chain reaction}

Real-time polymerase chain reaction was performed using Applied Biosystems 7500 machine. TaqMan-based primers for alkaline phosphatase (ALP; Hs 00758162_m1; NM_000478.3), bone sialoprotein (BSP; Hs 00173720_m1; NM_004967.3), matrix Gla protein (MGP; Hs 00179899_m1; NM_000900.2), and hyaluronan-mediated motility receptor (RHAMM; Hs 00234864_m1; NM_012484) were purchased from Applied Biosystems. Thirty-eight nanograms of RNA was used per each cDNA sample. Delta-Ct was calculated by the subtraction $\mathrm{Ct}$ value of the TATA box-binding protein (TBP) gene (NM 003194.3). The results for BPCs and differentiating MSCs were normalized to gene expression in undifferentiated MSCs.

\section{Animal studies}

Animal studies were performed under the approval of the Institutional Animal Care and Use Committee of the Tel Aviv University. An 5-mm-diameter defect was produced with a high-speed, water-cooled, diamond wheel-shaped bur in each side of the calvarial bone of inbred Fischer 344 rats. Care was taken not to injure the underlying brain tissue. Syngeneic BPCs seeded on scaffolds were transplanted into the right side. The left side was transplanted with the same type of scaffold, but without cells. Eight animals per each type of scaffold were transplanted. A control group of 4 animals was transplanted with the scaffold alone without cells on the right side, whereas the contralateral side remained untreated. The animals were euthanized at 6 weeks after transplantation. Skull tissues were subjected to Faxitron contact microradiography, then embedded in methyl methacrylate, and sectioned coronally at $4-\mu \mathrm{m}$ thickness roughly through the middle of the 2 defects. Sections were stained with Masson's TriChrome to identify the new bone in the defect area (dark blue). A rectangle of $1.5-\mathrm{mm}$ width was superimposed on the defect, and bone and total areas were measured. Percentage of the new bone in the defect was calculated. The study was double blinded.

\section{Statistical analysis}

Data are presented as mean \pm SD. Comparisons were made by using a two-tailed Student's $t$-test or a one-way analysis of variance for experiments with more than two subgroups. Probability values were considered statistically significant at $p<0.05$.

\section{Results}

\section{Human BPC yields}

The yield of BPCs p0 was $0.71 \pm 0.39 \times 10^{6}$ per $1 \mathrm{~mL} \mathrm{BM}$ $(n=23)$. When the BPC culture was continued for another week, the yield of BPCs was $1.05 \pm 0.36(n=13)$. The same $\mathrm{BM}$ batches were used for production of MSCs. MSCs were produced by plating $10 \times 10^{6} \mathrm{MNCs}$ (corresponded to $\sim 1 \mathrm{~mL} \mathrm{BM}$ ) in GM containing $20 \%$ FCS and by culturing adherent cells for 2 weeks (MSCs p0). The yield of MSCs p0 was $0.3 \pm 0.17 \times 10^{6}(n=21)$. Significantly, a lower yield of MSCs $(p=0.00043)$ suggested that conditions of the described BM culture are more favorable for stem cell/progenitor cell proliferation than commonly used MSC culture conditions.

\section{Osteogenic differentiation of human BPCs po}

When MSCs were induced to differentiate in an OM, the level of enzymatic activity of ALP gradually increased within 2 weeks (Fig. 1A). For production BPCs p0, unprocessed BM was also cultured in the OM for 2 weeks. ALP activity of the resulting BPCs was significantly higher than ALP activity of MSCs subjected to osteogenic differentiation for 1 week $\left(p<10^{6}\right)$ and for 2 weeks $(p<0.0025)$ (Fig. 1A). Gradual increase of ALP enzymatic activity in MSCs subjected to osteogenic differentiation paralleled the kinetics of its surface expression analyzed by flow cytometry. As illustrated by histograms in Figure 2B, most undifferentiated MSCs did not express ALP, although a small population was positive, probably due to spontaneous differentiation. At 1 week after addition of the OM, a significant number of MSCs expressed ALP, but a clear-cut population of ALP-negative cells still remained, and only by 2 weeks of differentiation, most of MSCs became positive. The flow cytometry analysis of ALP expression in BPCs p0 demonstrated a homogeneous ALP expression (a histogram at the bottom of Fig. 1B has practically a normal bell-shape distribution). The mean fluorescence intensity (MFI) of BPCs was higher than MFI of MSCs differentiated for 2 weeks; however, this difference was not statistically significant (Fig. 1C). 


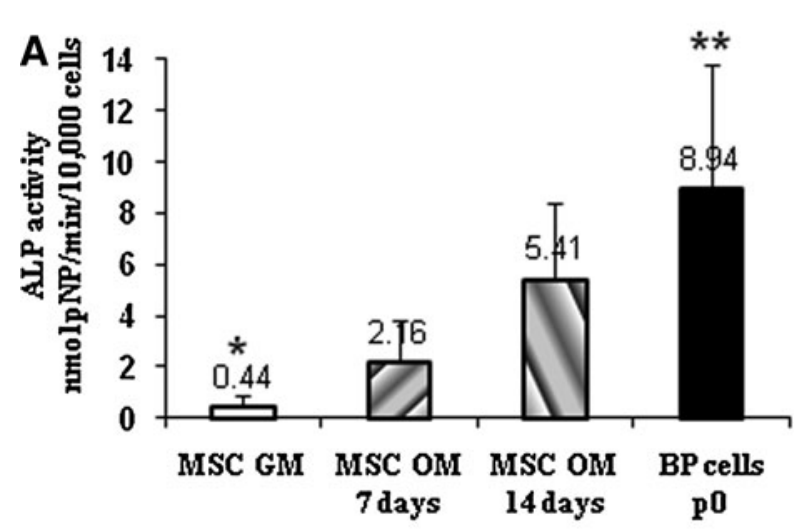

B
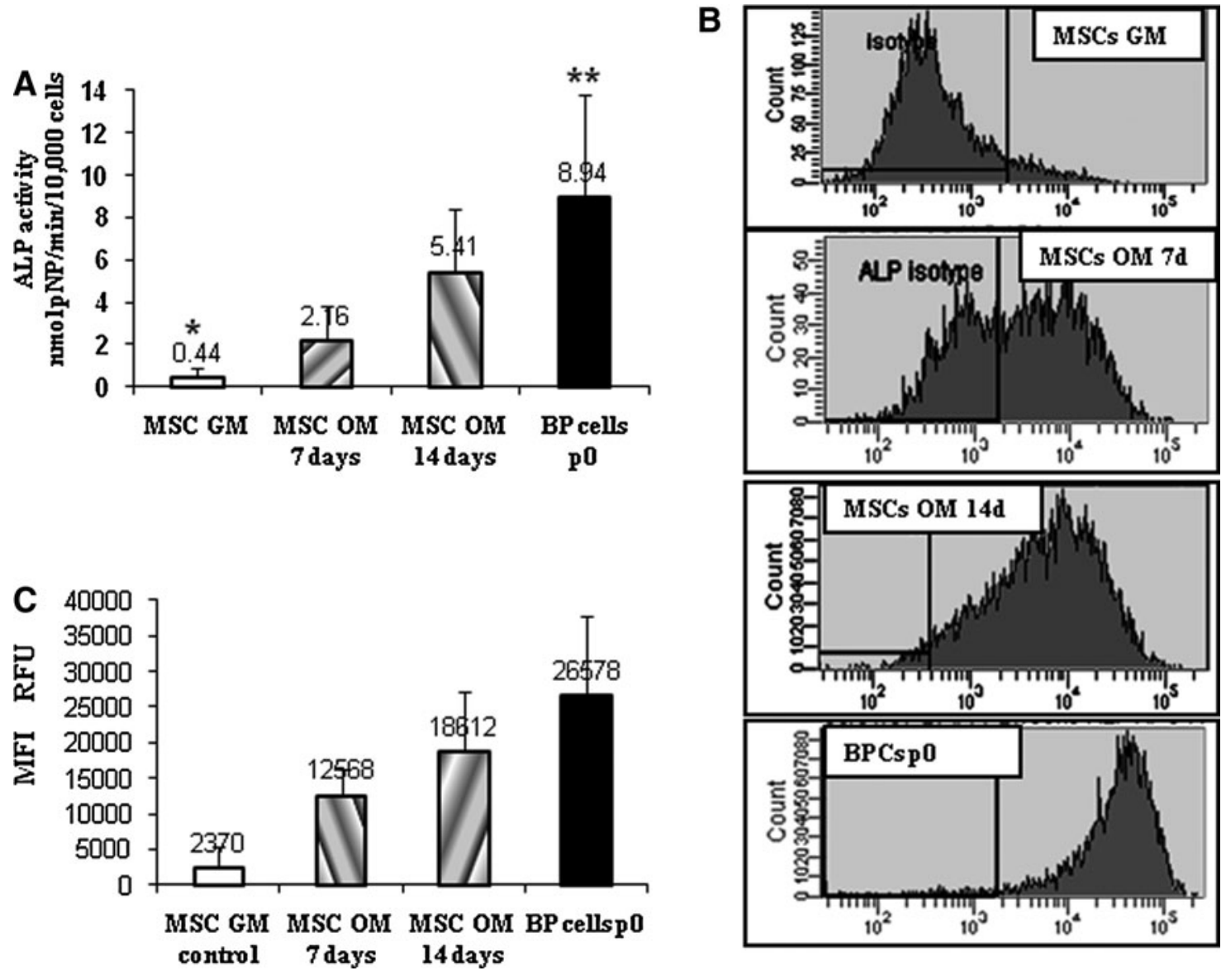

FIG. 1. Comparison of mesenchymal stem cells (MSCs) and bone progenitor cells (BPCs) derived from human bone marrow (BM). (A) Alkaline phosphatase (ALP) enzymatic activity ( $\mathrm{mmol} \mathrm{pNP} / \mathrm{min} / 10,000$ cells) in undifferentiated MSCs (MSCs GM); in MSCs incubated in an osteogenic medium (OM) for 7 days (MSCs OM 7 days) and for 14 days (MSCs OM 14 days); and in BPCs (BP cells p0); $n=10$. Statistically significant differences are indicated by asterisks. (B) Flow cytometry analysis of surface expression of ALP. Representative histograms for undifferentiated MSCs (top), MSCs differentiated for 7 and 14 days (middle), and BPCs p0 (bottom), derived from the same BM. Positions of the isotype control peaks are identified on histograms as black gate markers. (C) Flow cytometry analysis of surface expression of ALP. Mean fluorescence intensity (MFI) of cells stained with ALP antibodies; $n=5$.

\section{Production of human BPCs on scaffolds}

To demonstrate proliferation of BPCs on scaffolds, the scaffolds with BPCs p0 were washed and rotated in a fresh OM for additional 1-4 weeks (3-6 weeks from the initiation of culture). During the third week, the number of cells per scaffold practically doubled, although the proliferation rate of BPCs seeded on PLA/ceramic scaffolds was somewhat lower than that of BPCs seeded on PLA and PLA/PGA scaffolds (Fig. 2A). Extended culturing of BPCs on scaffolds resulted in practically an exponential growth (Fig. 2B). ALP activity of BPCs p0 growing on PLA and PLA/ceramic scaffolds was $8.8 \pm 2.4$ and $10.7 \pm 0.8 \mathrm{nmol} \mathrm{p}-\mathrm{NP} / \mathrm{min} / 10,000$ cells, respectively ( $n=3$ for each scaffold). BPC growth and differentiation are illustrated by Figure 3A, showing BPCs seeded on PLA/PGA 50/50 translucent scaffolds. Individual live cells stained with Calcein-AM could be seen in greater quantities at 6 weeks than at 2 weeks after initiation of BM culture. Histochemistry for ALP activity and for calcium deposits confirmed that proliferated BPCs retained their osteogenic character.
The osteogenic status of BPCs growing either on plastic or on scaffolds was also demonstrated on a gene level. All BPC samples were cultured for 2 weeks from the moment of plating of unprocessed BM in an OM. For comparison, MSCs derived from the same batches of BM were also differentiated in the OM for 2 weeks, although this time period might not be optimal for maximum expression of the studied genes. Both BPCs and differentiated MSCs had similar patterns of expression of osteogenic markers, although some of the genes (e.g., $A L P$ ) were upregulated to the higher extent in BPCs, whereas others genes such as bone sialoprotein $(B S P)$ and matrix Gla protein $(M G P)$ had higher levels of expression in MSCs differentiated for 2 weeks (Fig. 4A-F). The levels of expression of all tested genes were many folds higher in BPCs than in undifferentiated MSCs or in MSCs differentiated for 1 week. The fold increase of each gene over undifferentiated MSCs varied from batch to batch. A representative of three experiments is shown in Figure 4 . The error bars show variability of triplicates of the same RNA sample. Expression of the hyaluronan-mediated motility receptor (RHAMM) decreased many folds in MSCs upon differentiation and also in BPCs. (Fig. 4G, H). 
A

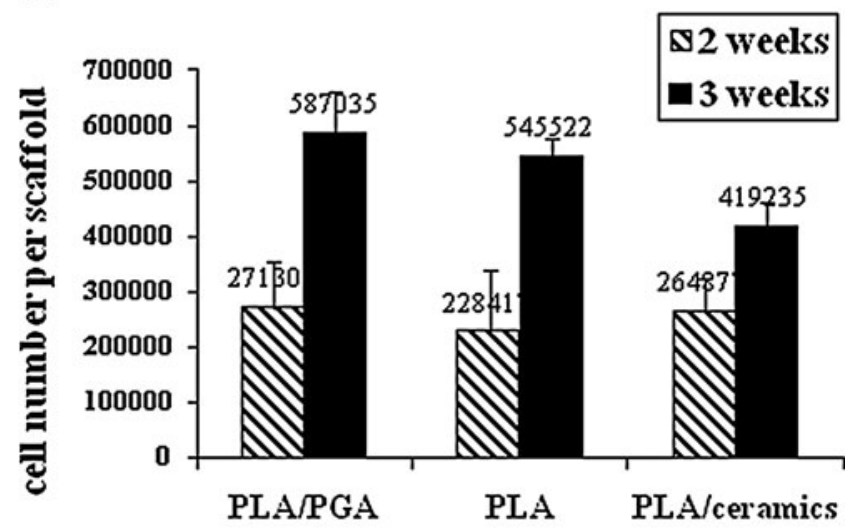

B

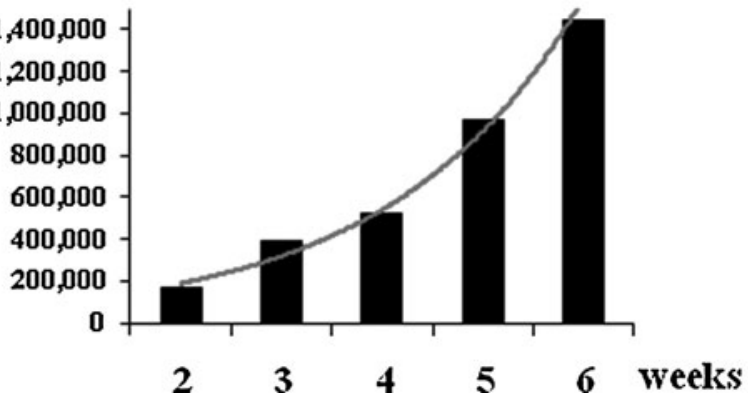

FIG. 2. Proliferation of human BP cells produced on scaffolds. (A) Human BPC yields on different scaffolds. PLApolylactic acid; PLA/PGA-polylactic/polyglycolic acid (50/50\%); PLA/ceramics-PLA compounded with $80 \%$ beta-tricalcium phosphate; $n=3-4$. (B) Proliferation of BPCs on PLA scaffolds. The results of one of two experiments are shown.

\section{Characterization of rat $B P C s$}

The data given in Table 1 demonstrate that while the yield of rat BPCs was significantly higher than the yield of human BPCs, their ALP activity and $\mathrm{Ca}^{2+}$ deposition were of the same order (also illustrated by microphotographs in Fig. 3C). Similar to human BPCs, rat BPCs populated the scaffolds. Rat BPCs seeded on translucent scaffolds are visualized with Calcein-AM (Fig. 3B, two upper images). Histochemistry staining demonstrated high ALP activity (Fig. 3B, bottom image).

\section{Effect of rat BPCs on bone repair in vivo}

According to the Alamar Blue assay, the number of BPCs on scaffolds used for transplantation was 95,140 $\pm 8,955$ for PLA/PGA (75/25); 71,607 $\pm 14,003$ for PLA/PGA (50/50); and $86,269 \pm 4,172$ for PLA/ceramic scaffolds $(n=5)$. As illustrated in Figures 5B and C, significant bone fill was seen in the right defect area in the majority of animals, 6 weeks after transplantation with PLA/PGA 50/50 and PLA/PGA 75/ 25 scaffolds.

Scattered calcified tissue on the left side is seen at the edge of the defect and not in the center. Defects transplanted with PLA/ceramic scaffolds had a radio-opaque material on both sides due to the presence of beta-tricalcium phosphate in the

scaffolds (Fig. 5D). No bone formation was observed in control animals (Fig. 5A), confirming that the defect of this size could not been naturally healed (critical-sized defect). The $\mathrm{X}$-ray results are summarized in Figure 6A. There was a statistically significant difference in the amount of bone in the defects transplanted with cells and those transplanted with a scaffold alone for both PLA/PGA 75/25 and 50/50 scaffolds, although somewhat less bone formation was observed in PLA/PGA 50/50 scaffolds; however, one animal demonstrated complete closure of the defect (animal 1, Fig. 5C). The results of histomorphometry (see examples of histological sections corresponding to X-ray images in Fig. 5) are summarized in Figure 6B. Histological examination of the defects transplanted with PLA/ceramic scaffolds demonstrated a significant difference between the defects transplanted with BPCs and the defects transplanted with the scaffold alone. The difference between the defects transplanted with PLA/ PGA 50/50 scaffolds seeded with BPCs and those without cells was not statistically significant due to the high variability between the animals.

\section{Discussion}

It has been hypothesized that adult stem cells are cells that emerge during development and then are somehow conserved in the adult organism for tissue/organ maintenance and repair. ${ }^{11,12}$ If such conservation occurs at various stages of development, then the resulting stem/progenitor cells should differ in the degree of maturity and differentiation potential. Heterogeneity of stem cells in the BM is reflected by the fact that various methods of isolation of BM-derived stromal cells yield stem cells with a different phenotype and differentiation potential. ${ }^{13-17}$ Perhaps, none of the current methods of MSCs isolation and expansion ${ }^{18}$ targets all BM stem cell resources. The most common method of isolation of MSCs from BM relies on the ability of MSCs to adhere to plastic. However, adherent cells are not homogenous and represent different precursor populations. ${ }^{19}$ For example, although osteogenic differentiation considered the default lineage of MSCs, only $60 \%$ of colonies obtained through adhesion selection form bone after transplantation in vivo. ${ }^{20}$ Humoral and intercellular signals within the BM microenvironment, such as vascular cells, pericytes, and hematopoietic stem cells, constituting a stem cell niche, control fate decisions of the mesenchymal progenitors. ${ }^{21-23}$ Destruction of this niche during MSC isolation might result in lower yields and suboptimal differentiation potential of isolated stem and progenitor cells.

On the basis of this knowledge, we attempted to produce predifferentiated osteoprogenitors from unprocessed BM bypassing isolation of MSCs. Human BM aspirates were incubated for 2 weeks with a commonly used OM, except no FCS, serum substitutes, or growth factors were added, because responding stem and/or progenitor cells were present in the BM milieu. Indeed, these culture conditions were more favorable for proliferation of stem/osteoprogenitor cells than culture conditions commonly used for derivation and expansion of MSCs, achieving higher yields of BPCs. Besides biological factors mentioned above, dexamethasone present in the OM could also have a mitogenic effect. ${ }^{24}$

Differentiation and seeding of BPCs on a scaffold occur simultaneously in a simple rotational bioreactor system. ${ }^{25}$ 
FIG. 3. Assessment of osteogenic differentiation of human and rat BPCs. (A) Human BPCs seeded on translucent PLA/PGA (50/ 50) scaffolds were analyzed at 2 weeks after BM plating (left) or after additional 4 weeks of culture in an OM (right). Top: Calcein-AM live-cell staining (green); Middle: Fast Blue RR staining for ALP activity (purple); Bottom: Alizarin red staining for calcium deposits (red). Magnification $\times 100$. (B) Rat BPCs seeded on PLA/ PGA (50/50) scaffolds were stained with Calcein-AM (top, magnification $\times 25$; middle, magnification $\times 100$ ), or with Fast Blue RR for ALP activity (bottom, purple, magnification $\times 100$ ). (C) Human (top) or rat (bottom) BPCs growing on plastic were stained with Fast Blue RR for ALP activity (left; purple) and with Alizarin red for calcium deposits (right; red). Magnification $\times 100$.
A

Live cell
Staining
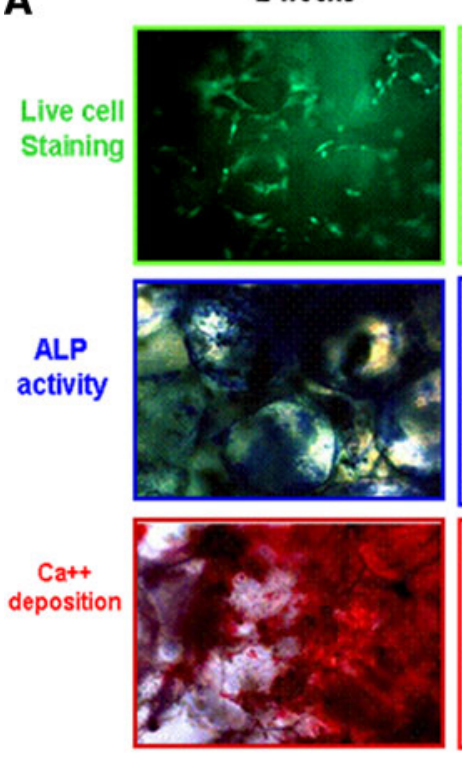

PLA/PGA 50/50
6 weeks
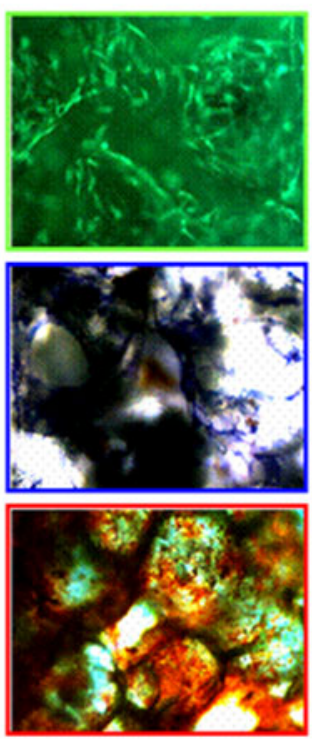

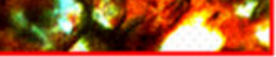

\section{(}

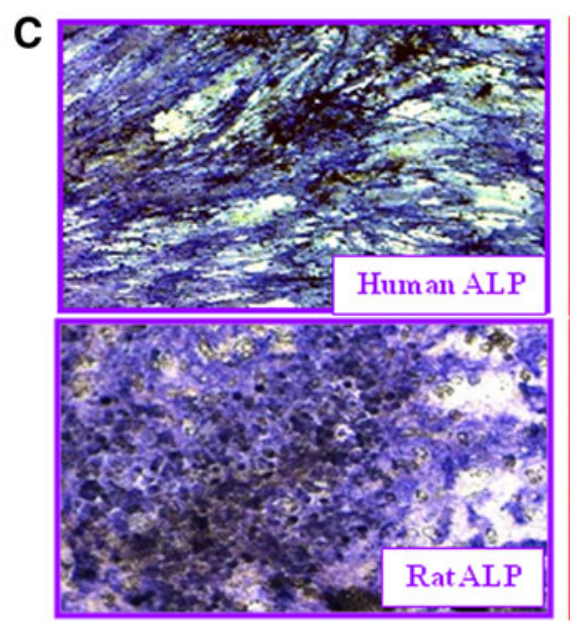

B
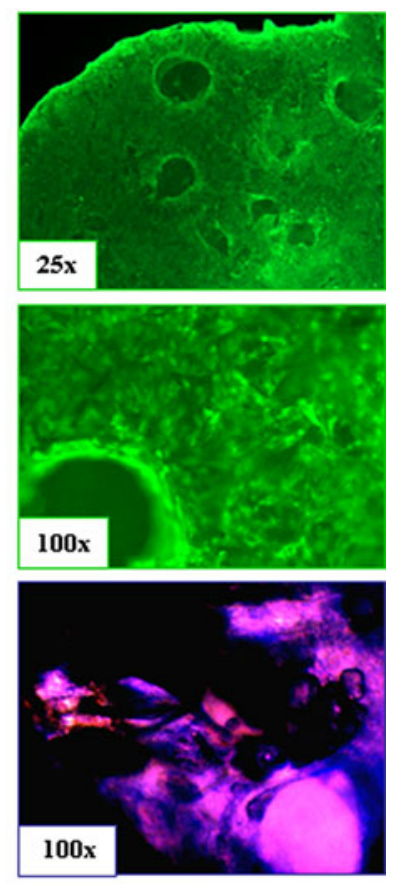

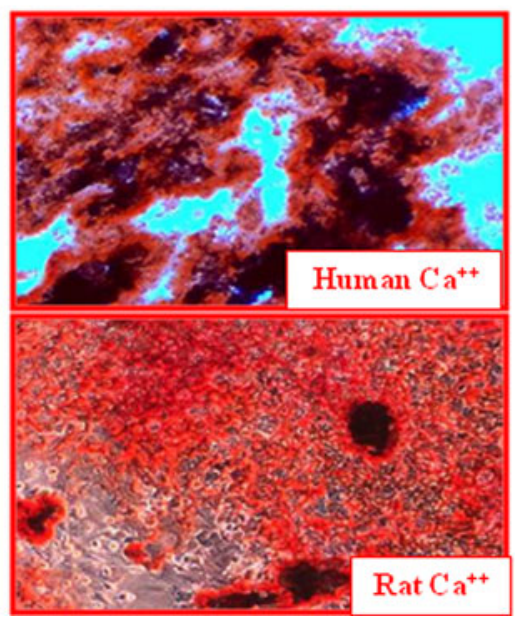

Based on the results shown in Figure 2A, the yield of BPCs on scaffolds could be estimated as 100,000-150,000 cells per $1 \mathrm{~mL}$ $\mathrm{BM}$. Because BPCs were produced by incubation in the OM for 2 weeks, their osteogenic status was compared to that of MSCs differentiated in a conventional OM for 2 weeks. MSCs were derived from the same batches of BM. BPCs produced by the new method were compatible with osteoprogenitors derived from MSCs as demonstrated by surface expression of ALP, ALP activity, $\mathrm{Ca}^{2+}$ deposition, and the levels of expression of osteogenic genes. Two genes known to be expressed during osteogenesis $A L P$ and $B S P^{26}$ were upregulated many folds in both BPCs and differentiated MSCs, although the absolute levels of upregulation varied as the time of their maximal expression was not optimized, but rather standardized to 2 weeks. Two other genes, MGP, a calcium-binding protein similar to osteocalcin, ${ }^{27}$ and RHAMM, a hyaluronan receptor similar to the main hyaluronan receptor, $\mathrm{CD} 44,{ }^{28}$ were studied in our laboratory as po- tential new markers of osteogenic differentiation. Indeed, MGP was upregulated up to 50-fold upon differentiation of MSCs. A 10-fold upregulation of MGP was also demonstrated in BPCs. Surprisingly, expression of RHAMM drastically decreased upon osteogenic differentiation of MSCs. Similarly, RHAMM expression in BPCs was many folds lower than in undifferentiated MSCs.

Osteogenic potential of BPCs seeded on scaffolds was tested in vivo in an autologous critical-sized calvarial defect model. ${ }^{29}$ Quantitative radiographic (Faxitron) analysis and histomorphometry demonstrated larger amount of bone in the defects transplanted with scaffolds seeded with BPCs compared to scaffolds without cells. No direct comparison with MSCs was made because of the variability of the methods for making tissue-engineering grafts seeded with MSCs. Cell-plating time, seeding density, and preseeding or postseeding differentiation influence the bone repair efficacy of such constructs, making their comparison to BPC-seeded scaffolds unreliable. 

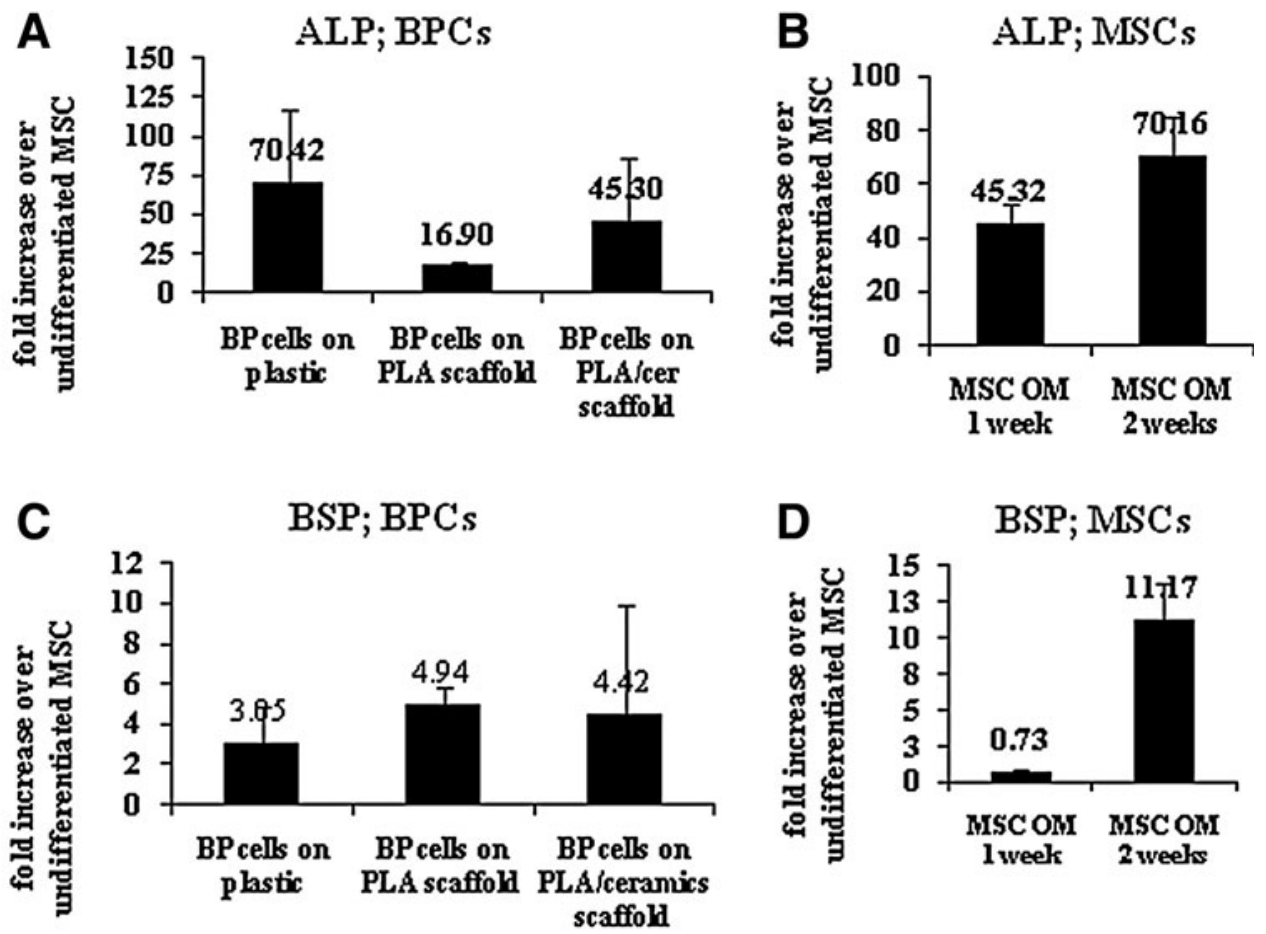

FIG. 4. Osteogenic gene expression in human BPCs and in differentiating MSCs. (A, C, E, G) BPCs p0 at 2 weeks after plating BM. (B, D, F, H) MSCs isolated from the same BM and differentiated in an OM for 1 and 2 weeks. All results were normalized to the gene expression levels in undifferentiated MSCs. (A, B) Alkaline phosphatase
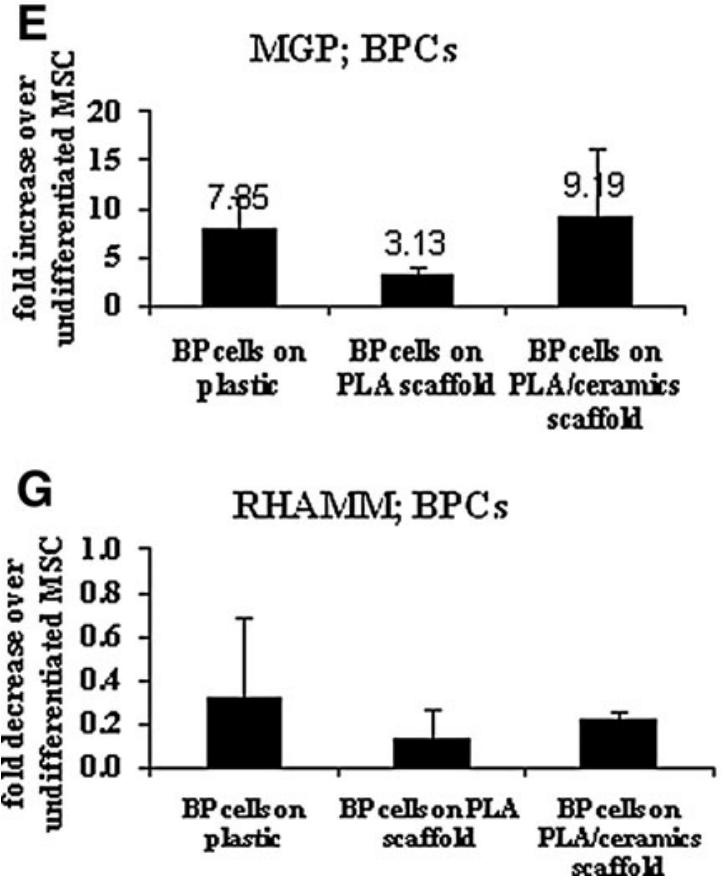

$\mathbf{F}$
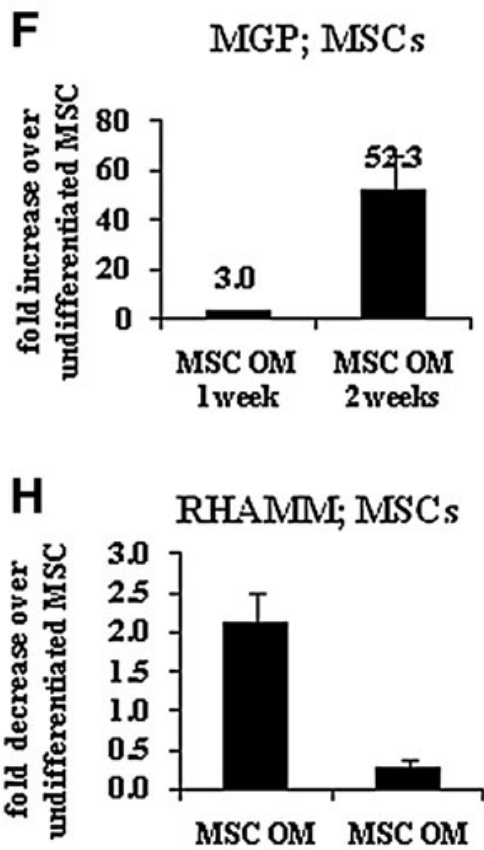

(ALP); (C, D) bone sialoprotein (BSP); (E, F) Matrix Gla Protein (MGP); $(G, H)$ Hyaluronan-mediated motility receptor (RHAMM). Each bar is mean \pm SD of triplicates for one BM batch. A representative of 3 experiments with different BM batches is shown.
However, the percentage of a new bone $(76.1 \% \pm 18.3 \%)$ in the defects transplanted with PLA/PGA scaffolds seeded with about 100,000 BPCs was compatible to or even higher than in published studies of bone repair by MSCs in the calvarial model in mice $(50,000$ MSCs transplanted on PLA/PGA scaffolds ${ }^{30}$ or $10^{7}$ MSCs in platelet-rich plasma $[\mathrm{PRP}]^{31}$ ), in rats (500,000 MSCs in agarose ${ }^{32}$ or umbilical cord blood-derived predifferentiated MSCs mixed with a demineralized bone matrix $^{33}$ or 300,000 MSCs in chitosan ${ }^{34}$ ), and in rabbits, where $2 \times 10^{7}$ MSCs were transplanted in PRP gel. In the latter study, MSCs predifferentiated with dexamethasone produced better results than undifferentiated MSCs. ${ }^{35}$

The amount of bone observed by X-ray evaluation was higher than shown by the histological analysis, when compared on a per-animal basis. This could be explained by several factors: (1) the X-ray quantitation refers to the whole defect, whereas the histology refers only to a $5-\mu \mathrm{m}-$ thick band roughly at the center of the defect. Therefore, any radio-opaque substance at the lower or upper parts of the defect (as seen on the radiograph) will not be 
Table 1. Characterization of Human and Rat Bone Progenitor Cells Derived from Intact Bone Marrow

\begin{tabular}{lccccc}
\hline Species & BPC yield (per $m L$ BM) & ALP activity $($ nmol p-NP/min per 10,000 cells) & Ca2 + deposition $(\mu g / 10,000$ cells) \\
\hline Human & $0.7 \pm 0.4 \times 10^{6}$ & $(n=23)$ & $8.9 \pm 4.9$ & $(n=10)$ & $5.42 \pm 3.0$ \\
Rat & $1.2 \pm 0.5 \times 10^{6}$ & $(n=6)$ & $13.4 \pm 2.4$ & $(n=6)$ & $2.5 \pm 1.1$ \\
\hline
\end{tabular}

BPC, bone progenitor cell; BM, bone marrow; ALP, alkaline phosphatase.

measured by the histomorphometry. This inherent discrepancy will result in underestimation of the mineralized material by the histomorphometry and a better, more global assessment by the radiographic analysis; (2) on the other hand, the radiographic analysis does not discriminate between new bone formed as part of the healing process, bone chips that were left within the defect during surgery, and mineralized scaffolds (all 3 forms were found in the histological sections). Therefore, the radiographic data will usually give an overestimation of the amount of new bone. The histological inspection is the only means to ascertain the formation of true new bone, and thus a healing process. Combination of the two methods yielded a quite accurate report of the outcome of the animal studies and
A
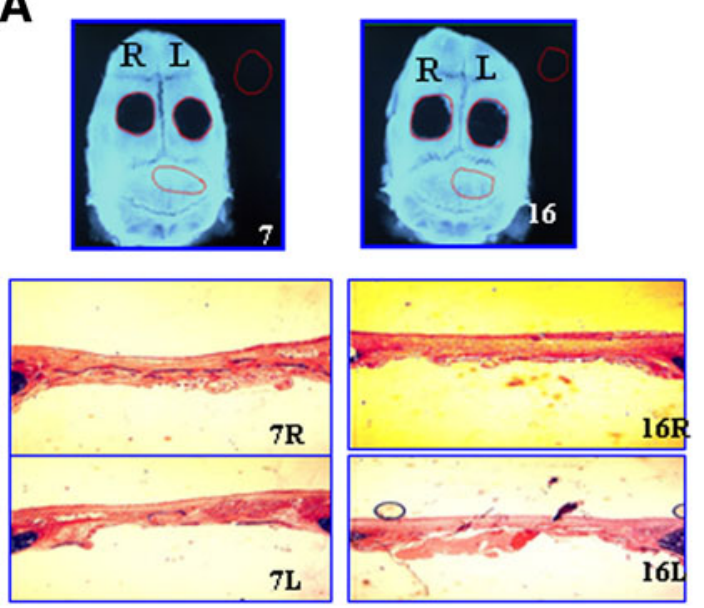

C

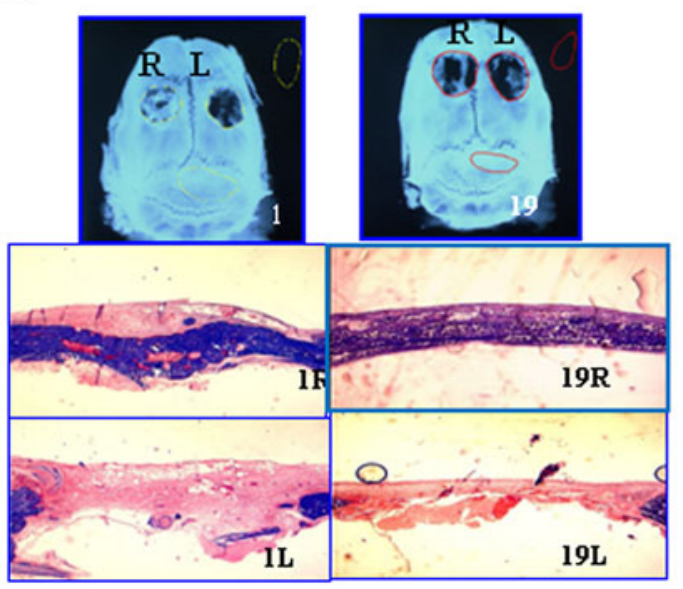

B
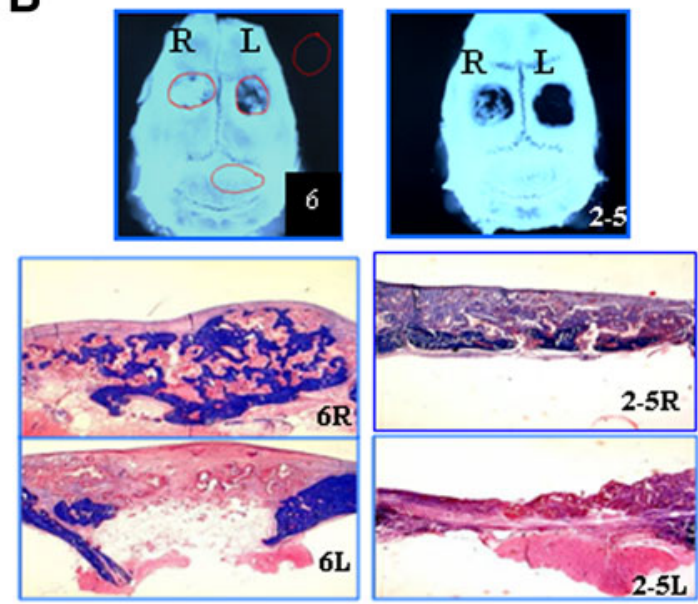

D

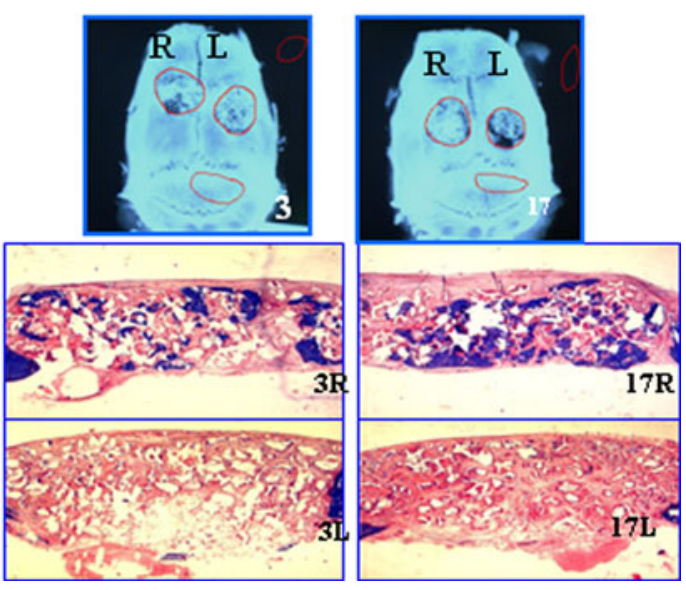

FIG. 5. Transplantation of rat BPCs in calvarial defects. Representative radiographs (top) and corresponding histological sections of the defect area (bottom). R, right side transplanted with a scaffold seeded with cells; L, left side transplanted with the same scaffold with no cells. The numbers in the images represent the identification numbers of the animals. Red circles identify the bone area used for the OD calibration of Faxitron; OD of the no bone area (black) was calibrated on the empty X-Ray film (not shown). (A) Control group transplanted with PLA/PGA 75/25\% scaffold without cells on the right (R) side; the defect on the left (L) side was left empty. (B) PLA/PGA 75/25\% group with BP cells on the right (R), scaffold alone on the left (L). (C) PLA/PGA 50/50\% group with BP cells on the right (R), scaffold alone on the left $(\mathrm{L})$, and (D) PLA/ceramic group with BP cells on the right (R), scaffold alone on the left (L). 
A

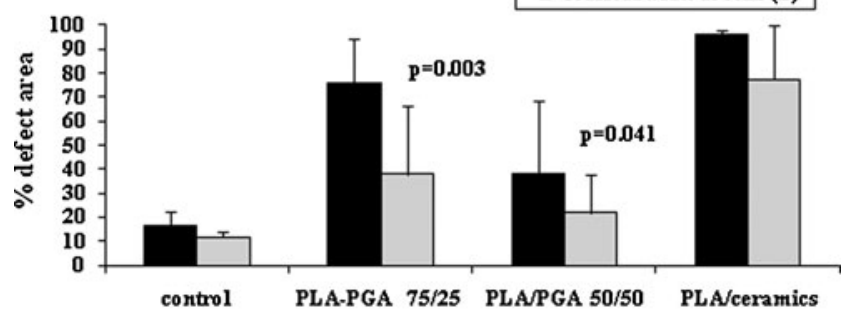

B

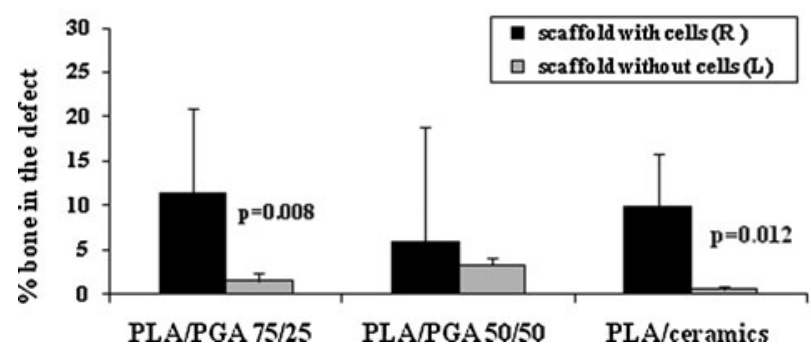

FIG. 6. Summarized results of BPC transplantation. (A) Percentage of the bone area in the defect based on an X-ray analysis. (B) Percentage of the bone area in the defect based on histological examination.

demonstrated that BPCs produced by direct osteogenic differentiation of intact BM are capable of generation of a new bone in vivo.

\section{Acknowledgments}

The experimental work presented in the article was performed while I.G., N.A., and M.S. were working at Teva Pharmaceutical Industries, Petach Tikva, Israel. The authors want to thank Teva administration for their financial support of the study.

\section{Author Disclosure Statement}

No competing financial interests exist.

\section{References}

1. Finkemeier CG. Bone-grafting and bone-graft substitutes. J Bone Joint Surg Am. 2002;84-A:454-464.

2. Caplan AI. Review: mesenchymal stem cells: cell-based reconstructive therapy in orthopedics. Tissue Eng. 2005;11:1198-1211.

3. Guldberg RE, Oest ME, Dupont K, et al. Biologic augmentation of polymer scaffolds for bone repair. J Musculoskelet Neuronal Interact. 2007;7:333-334.

4. Arthur A, Zannettino A, Gronthos S. The therapeutic applications of multipotential mesenchymal/stromal stem cells in skeletal tissue repair. J Cell Physiol. 2009;218:237-245.

5. Beyth S, Schroeder J, Liebergall M. Stem cells in bone diseases: current clinical practice. Br Med Bull. 2011;99:199-210.

6. Bonab MM, Alimoghaddam K, Talebian F, et al. Aging of mesenchymal stem cell in vitro. BMC Cell Biol. 2006;7:14.

7. Zhou YF, Bosch-Marce M, et al. Spontaneous transformation of cultured mouse bone marrow-derived stromal cells. Cancer Res. 2006;66:10849-10854.
8. Grayson WL, Bhumiratana, S, Grace Chao PH, et al. Spatial regulation of human mesenchymal stem cell differentiation in engineered osteochondral constructs: effects of pre-differentiation, soluble factors and medium perfusion. Osteoarthritis Cartilage. 2010;18: 714-723.

9. Halfon S, Abramov N, Grinblat B, et al. Markers distinguishing mesenchymal stem cells from fibroblasts are downregulated with passaging. Stem Cells Dev. 2011;20:53-66.

10. Colter DC, Class R, DiGirolamo CM, et al. Rapid expansion of recycling stem cells in cultures of plastic-adherent cells from human bone marrow. Proc Natl Acad Sci USA. 2000;97:3213-3218.

11. da Silva Meirelles L, Chagastelles PC, Nardi NB. Mesenchymal stem cells reside in virtually all post-natal organs and tissues. J Cell Sci. 2006;119:2204-2213.

12. Ratajczak MZ, Kucia M, Majka M, et al. Heterogeneous populations of bone marrow stem cells-are we spotting on the same cells from the different angles? Folia Histochem Cytobiol. 2004;42:139-416.

13. Bianco P, Robey, PG. Marrow stromal stem cells. J Clin Invest. 2000;105:1663-1668.

14. Colter DC, Sekiya I, Prockop DJ. Identification of a subpopulation of rapidly self-renewing and multipotential adult stem cells in colonies of human marrow stromal cells. Proc Natl Acad Sci USA. 2001;98:7841-7845.

15. D'Ippolito G, Diabira S, Howard GA, et al. Marrow-isolated adult multilineage inducible (MIAMI) cells, a unique population of postnatal young and old human cells with extensive expansion and differentiation potential. J Cell Sci. 2004; 117(Pt 14):2971-2981.

16. Kucia M, Ratajczak J, Ratajczak, MZ (2005). Bone marrow as a source of circulating CXCR4 + tissue-committed stem cells. Biol Cell. 2005;97:133-146.

17. Sohni A, Verfaillie CM. Multipotent adult progenitor cells. Best Pract Res Clin Haematol. 2011;24:3-11.

18. Vemuri MC, Chase LG, Rao MS. Mesenchymal stem cell assays and applications. Methods Mol Biol. 2011;698:3-8.

19. Smith JR, Pochampally R, Perry A, et al. Isolation of a highly clonogenic and multipotential subfraction of adult stem cells from BM stroma. Stem Cells. 2004;22:823-831.

20. Kuznetsov SA, Krebsbach PH, Satomura K et al. Singlecolony derived strains of human marrow stromal fibroblasts form bone after transplantation in vivo. J Bone Miner Res 1997;12:1335-1347.

21. Bianco P. Minireview: the stem cell next door: skeletal and hematopoietic stem cell "niches" in bone. Endocrinology. 2011;152:2957-2962.

22. Augello A, Kurth TB, De Bari C. Mesenchymal stem cells: a perspective from in vitro cultures to in vivo migration and niches. Eur Cell Mater. 2010;20:121-133.

23. Di Nardo P and Parker GC. Stem cell standardization. Stem Cells Dev. 2011;20:375-377.

24. Kuznetsov SA, Mankani MH, Robey PG. In vivo formation of bone and haematopoietic territories by transplanted human bone marrow stromal cells generated in medium with and without osteogenic supplements. J Tissue Eng Regen Med. 2011. [Epub ahead of print]; DOI: 10.1002/term.515.

25. Gorodetsky R, Clark RA, An J, et al. Fibrin microbeads (FMB) as biodegradable carriers for culturing cells and for accelerating wound healing. J Invest Dermatol. 1999;112:866-872.

26. Frank O, Heim M, Jakob M, et al. Real-time quantitative RTPCR analysis of human bone marrow stromal cells during osteogenic differentiation in vitro. J Cell Biochem. 2002;85:737746. 
27. Vattikuti R, Towler DA. Osteogenic regulation of vascular calcification: an early perspective. Am J Physiol Endocrinol Metab. 2004;286:E686-E696.

28. Naor D, Nedvetzki S, Walmsley M, et al. CD44 involvement in autoimmune inflammations: the lesson to be learned from CD44-targeting by antibody or from knockout mice. Ann N Y Acad Sci. 2007;1110:233-247.

29. Artzi Z, Kozlovsky A, Nemcovsky CE, et al. Histomorphometric evaluation of natural mineral combined with a synthetic cell-binding peptide (P-15) in critical-size defects in the rat calvaria. Int J Oral Maxillofac Implants. 2008; 23:1063-1070.

30. Cowan CM, Shi YY, Aalami OO, et al. Adipose-derived adult stromal cells heal critical-size mouse calvarial defects. Nat Biotechnol. 2004;22:560-567.

31. Monteiro BS, Del Carlo RJ, Argôlo-Neto NM, et al. Association of mesenchymal stem cells with platelet rich plasma on the repair of critical calvarial defects in mice. Acta Cir Bras. 2012;27:201-209.

32. Osugi M, Katagiri W, Yoshimi R, et al. Conditioned media from mesenchymal stem cells enhanced bone regeneration in rat calvarial bone defects. Tissue Eng Part A. 2012. [Epub ahead of print]; DOI: 10.1089/ten.tea.2011.0325
33. Liu GP, Li YL, Sun J, et al. [Repair of calvarial defects with human umbilical cord blood derived mesenchymal stem cells and demineralized bone matrix in athymic rats]. Zhonghua Zheng Xing Wai Ke Za Zhi. 2010;26:3438 (abstract).

34. Stephan SJ, Tholpady SS, Gross B, et al. Injectable tissueengineered bone repair of a rat calvarial defect. Laryngoscope. 2010;120:895-901.

35. Jiang ZQ, Liu HY, Zhang LP, et al. Repair of calvarial defects in rabbits with platelet-rich plasma as the scaffold for carrying bone marrow stromal cells. Oral Surg Oral Med Oral Pathol Oral Radiol Endod. 2012;113:327-333.

Address correspondence to: Irene Ginis, $M D, P h D$ Teva Pharmaceutical Industries LTD

5 Basel Street

Petach Tikva 49131

Israel

E-mail: irene@macrocure.com; iginis1@gmail.com 